\title{
Perturbation of the Earth's rotation by monochromatic gravitational waves from astrophysical sources
}

\author{
Séverine Rosat ${ }^{1, *}$ and Josipa Majstorović ${ }^{2}$ \\ ${ }^{1}$ Institut Terre et Environnement de Strasbourg UMR 7063, \\ Université de Strasbourg/EOST, CNRS, 5 rue Descartes, 67084 Strasbourg, France \\ ${ }^{2}$ Université Grenoble Alpes, CNRS, ISTerre, 38000 Grenoble, France \\ (Received 9 December 2019; revised 15 December 2020; accepted 20 April 2021)
}

(PHYSICAL REVIEW D)

(Https://doi.org/10.1103/PhysRevD.103.104052)

\begin{abstract}
Gravitational waves (GWs) of astrophysical origin were detected for the first time in 2015 through strain deformation measured at the Earth's surface. The inertia tensor of the deformable Earth is also disturbed resulting in the perturbation of its rotation vector and excitation of the rotational normal modes. Using a linearized theory of gravitation and the linearized equations of conservation of the angular momentum, we compute the equatorial polar motion and length of day changes generated by GWs. We show that GWs of strain amplitude $h_{0}$ and frequency $f_{g}$ give rise to perturbations of the inertia tensor of the Earth with an amplitude of $10^{14} h_{0} f_{g}^{2}$, resulting in relative perturbation of the Earth's rotation rate and equatorial polar motion respectively of the order $10^{6} h_{0} f_{g}^{2}$ and $10^{14} h_{0} f_{g}^{2}$. The amplitude of the rotational effect is much smaller than the geophysicallyinduced rotational perturbation even if a resonance with the Earth's rotational normal modes would be possible. The amplitude of this rotational effect increases with the frequency but is several orders of magnitude below the theoretical sensitivity level of current geodetic instruments. The centrifugal deformation associated with the GW-induced polar motion would be $\sim 10^{6} f_{g}^{2} h_{0} \sim 10^{-17} / \sqrt{\mathrm{Hz}}$ for $f_{g}=10^{-4} \mathrm{~Hz}$ and $h_{0}=10^{-16}$. The strain amplitudes of such centrifugal deformation are beyond the detectability of current laser strainmeters used to detect GWs. In the future, improvement in the sensitivities of geophysical instruments to measure Earth's rotation fluctuations, particularly at sub-daily periods, and the development of the Laser Interferometer Space Antenna would make the present quantifications worth considering.
\end{abstract}

Keywords: gravitational wave detection, Earth's rotation

Gravitational waves (GWs) carry fundamental information on the time-space evolution of the Universe and its origin. Quests for detecting these gravitational waves of astrophysical origin have been numerous until the first detection in 2015 in the frame of the international scientific LIGO and Virgo collaborations [1]. GWs induce distortions of time-space causing strain deformations at the Earth's surface. Current laser interferometers like LIGO and Virgo can measure strain deformations at the Earth's surface with a precision of the order of $10^{-22}$ at frequencies around $100 \mathrm{~Hz}$. The signal detected on September, 14th 2015 is a transient signal of maximum amplitude $10^{-21}$ in strain and lasted about 0.15 second in a frequency band ranging from $35 \mathrm{~Hz}$ to $250 \mathrm{~Hz}$. This signal resulted from the coalescence of two black holes located at more than one billion of light years from the Earth. Since then there has been 11 detections in first (O1) and second (O2) observational runs [2], and 39 in the first part of third observational run (O3a) [3] bringing in total $50 \mathrm{GW}$ events to the present GW catalog (O1: September 2015 - January 2016; O2: November 2016 - August 2017; O3: April 2019 - September 2019). This catalog contains sources of different astrophysical parameters associated with binary black holes mergers, binary neutron stars, and neutron star black hole binaries.

Laser strainmeters are currently the foremost detectors of GWs. They are able to reach strain sensitivity of $10^{-23} / \sqrt{\mathrm{Hz}}$ (around $100 \mathrm{~Hz}$ ) in high frequency range $\left(10-10^{3} \mathrm{~Hz}\right.$ ). In the same range the resonant mass detectors has also been developed. Originally proposed by Weber [4] they consist in detecting the resonance effect with normal modes of the system induced by the gravitational waves. There are cylindrical cryogenic resonance devices with a characteristic strain sensitivity of $10^{-19}$ [5-7] and spherical resonant detectors such as miniGRAIL [8], the Mario Schenberg detector [9] that reach sensitivity of $10^{-20}$. At low frequencies $\left(10^{-4}-0.1 \mathrm{~Hz}\right)$ the most promising detectors are space-based Michelson-like interferometers, such as the Laser Interferometer Space Antenna (LISA) [10], designed to reach characteristic strain sensitivity of $10^{-20}$. Beside space-born detectors, more accessible ground-based torsion bar pendulum have been proposed with the target sensitivity set to $10^{-19} / \sqrt{\mathrm{Hz}}$ (at $0.1 \mathrm{~Hz}$ ) [11]. Some other proposal of 
ground-based detectors include also superconducting levitated masses [12] with possibility to reach a strain sensitivity of $10^{-19} / \sqrt{\mathrm{Hz}}$ (at $0.2 \mathrm{~Hz}$ ), and atomic interferometers [13] designed to reach peak strain sensitivity of $10^{-13} / \sqrt{\mathrm{Hz}}$ (at $2 \mathrm{~Hz}$ ). In very low frequency band the used detectors are pulsar timing arrays that measure the variations in time arrivals of the pulsar signals. The lower threshold of a characteristic sensitivity is set to $10^{-16}$ [14]. However, for the moment, any of these devices have not enabled the detection of GWs.

Like any mechanical system, the Earth possesses eigenmodes. When GWs are emitted, the local space-time of the Earth is deformed, perturbing the inertia tensor of the Earth and exciting elastic waves. An excitation of the Earth's normal modes would be possible and a resonance effect could occur. The idea to use the Earth as a GW receiver was already proposed by Dyson [15], Tuman [16], Jensen [17], Ben-Menahem [18] and more recently Coughlin and Harms [19] and Majstorović et al. [20, 21]. Those authors investigated the coupling of GWs with seismic modes of the Earth, hence at frequencies in the millihertz frequency band or at $1 \mathrm{~Hz}$, that is at frequencies lower than the operating frequency range of LIGO-Virgo instruments. Coughlin and Harms [19] proposed an upper limit of GW energy density at seismic frequencies, where sources of GWs can be for instance massive binaries or massive inspiraling black holes $[22,23]$. Also, the GW sensitivity limit in terms of global network of broadband seismometers [24], as well as Apollo Seismic Array [25] has been investigated. Weber [4] computed the irregular fluctuations in the Earth's rotation rate caused by an incident gravitational wave with a continuous spectrum. He showed that the induced perturbation would be too small to use the Earth's rotation as a detector based on the gravitational flux that would be required to explain Earth's rotational anomalies.

Other aspects of the Earth's rotation have been envisaged to detect GWs: that are using the Sagnac effect associated with the magnetic part of the Weyl tensor [26, 27].

In this paper, we consider the possibility to use the Earth's elasticity and its rotation as a detector for GWs. We quantify for the first time the perturbation of the Earth's instantaneous rotation axis in terms of spin rate and polar motion perturbations induced by GWs and their resonance with the Earth's rotational normal modes. The Chandler wobble (CW) represents a 432-day wobble of the Earth's rotation axis with respect to the figure axis [e.g. 28]. At the periods of the rotational CW mode, main sources of GWs could be supermassive binaries [29]. When considering an Earth's model with an elliptical fluid core, another mode exists: the free core nutation (FCN) which represents a diurnal wobble of the elliptical fluid core with respect to the mantle, in a terrestrial reference frame and a 432-day retrograde nutation as viewed from a celestial reference frame. The FCN mode is best determined by space nutation observations [e.g. 30]. When considering the presence of a solid inner core in the Earth's fluid core, two additional modes of rotation exist: the inner-core wobble and the free-inner core nutation which are the equivalent $\mathrm{CW}$ and FCN for the inner-core. These two modes have never been directly observed because of their much smaller effects. Consequently, we will ignore them. In the following, we first compute the perturbation of the inertia tensor exerted by GWs on the Earth. Then we use the linearized form of the conservation of the angular momentum equations to describe the transfer function of the Earth. Finally we provide some estimates of the rotation perturbations induced by GWs passing through the Earth in terms of polar motion and length of day changes that we compare with current instrumental sensitivities and geophysical excitations of the Earth's rotation.

\section{EFFECT OF A GRAVITATIONAL WAVE ON THE EARTH'S INERTIA TENSOR}

Far from massive sources of gravitational waves, GWs can be approximated as plane waves propagating in a flat space-time. In the weak-field linear approximation of gravity, the gravitational field can be considered as a perturbation of flat space-time and the field is static. The gravitational field is hence expressed as the decomposition of the metric into the Minkowski metric plus a small perturbation noted $h_{\mu \nu}$. In the following we are dropping Einstein summation convention and follow the notations used in Ben-Menahem [18] and Majstorović et al. [20]. The tensor $\mathbf{h}$ corresponds to the metric perturbation tensor as defined in the post-Newtonian approximation [22]. We define a GW traveling at the velocity of light $c$ by its amplitude $h_{0}$, its unit wave vector $\vec{p}$ normal to the wave front and its unit polarization tensor $\boldsymbol{\epsilon}$. The polarization tensor $\boldsymbol{\epsilon}$ of a GW is usually described as a function of two vectors $\mathbf{e}_{+}$and $\mathbf{e}_{\times}$defined as

$$
\boldsymbol{e}_{+}=\overrightarrow{\boldsymbol{l}}^{\mathrm{T}}-\overrightarrow{\boldsymbol{m}} \overrightarrow{\boldsymbol{m}}^{\mathrm{T}}=\left(\begin{array}{ccc}
1 & 0 & 0 \\
0 & -1 & 0 \\
0 & 0 & 0
\end{array}\right), \quad \boldsymbol{e}_{\times}=\overrightarrow{\boldsymbol{l}} \overrightarrow{\boldsymbol{m}}^{\mathrm{T}}+\overrightarrow{\boldsymbol{m}} \overrightarrow{\boldsymbol{l}}^{\mathrm{T}}=\left(\begin{array}{lll}
0 & 1 & 0 \\
1 & 0 & 0 \\
0 & 0 & 0
\end{array}\right) .
$$

We define the vector $\vec{p}$ along the unit vector $\overrightarrow{\boldsymbol{e}_{\boldsymbol{k}}}$. The unit vectors $\overrightarrow{\boldsymbol{l}}, \overrightarrow{\boldsymbol{m}}$ are related to the units of spherical coordinate system $\overrightarrow{\boldsymbol{e}}_{e}, \overrightarrow{\boldsymbol{e}}_{\lambda}$ through relations

$$
\begin{aligned}
\overrightarrow{\boldsymbol{l}} & =\cos \nu\left(-\overrightarrow{\boldsymbol{e}}_{e}\right)+\sin \nu\left(-\overrightarrow{\boldsymbol{e}}_{\lambda}\right), \\
\overrightarrow{\boldsymbol{m}} & =\sin \nu\left(\overrightarrow{\boldsymbol{e}}_{e}\right)+\cos \nu\left(-\overrightarrow{\boldsymbol{e}}_{\lambda}\right),
\end{aligned}
$$


with $e, \lambda$ and $\nu$ being three angles specifying the plane of polarization in $O$ (cf. Fig. (1)).

We note $\omega_{g}$ the angular frequency and $\vec{k}_{g}=\frac{\omega_{g}}{c} \vec{p}$ the wave vector. The metric tensor perturbation is

$$
\mathbf{h}=h_{i j}=h_{0} \boldsymbol{\epsilon} e^{i\left(\omega_{g} t-\vec{k}_{g} \cdot \vec{r}\right)} .
$$

The standard definition of the inertia tensor of a mass body of volume $V$ and density $\rho$ is given by

$$
I_{i j}=\int_{V} \rho\left(r^{2} \delta_{i j}-x_{i} x_{j}\right) d v,
$$

where $\delta$ is the Kronecker symbol and $r$ is the distance to the center of mass.

If we note $A$ and $C$ the main inertia moments of the ellipsoidal Earth and $c_{i j}$ the small perturbations, the inertia tensor is written

$$
I_{i j}=\left(\begin{array}{ccc}
A+c_{11} & c_{12} & c_{13} \\
c_{12} & A+c_{22} & c_{23} \\
c_{13} & c_{23} & C+c_{33}
\end{array}\right) \text {, }
$$

where we have assumed that the $c_{i j}$ are symmetric. The perturbation of the inertia tensor is trace-free so that $c_{11}+c_{22}+c_{33}=0$.

The main effect of GWs is to cause a quadrupole distortion of space-time or tidal forces. In such an external tidal field, the body will in response develop a trace-free quadrupolar moment $Q_{i j}$ defined in the Newtonian limit by $[22,31]$

$$
Q_{i j}=\int_{V} \rho\left(x_{i} x_{j}-\frac{1}{3} r^{2} \delta_{i j}\right) d v .
$$

We can relate the body quadrupole moment and the inertia tensor by

$$
Q_{i j}=\frac{\operatorname{tr}(I)}{3} \delta_{i j}-I_{i j},
$$

where $\operatorname{tr}(I)$ is the trace of $I$. We are interested in the perturbations of this moment (the departures from the principal moment of inertia $\mathrm{A}$ and $\mathrm{C}$ ) which are indeed directly related to the $c_{i j}$ by

$$
Q_{i j}=-c_{i j} .
$$

We consider a local Lorentz frame in which the GW is introduced as a tidal field. Using the fact that the Riemann tensor is invariant under the gauge transformation, we can evaluate it in the transverse traceless gauge. A GW corresponds to periodic changes in the Riemann curvature tensor that lead to deformations of a matter body through which the GW is passing. In the limit where the source of external quadrupolar tidal field is very far away, the induced changes in the inertia tensor $c_{i j}$ or quadrupole moment $Q_{i j}$ are proportional to the tidal field through the degree-two Love number $k_{2}$ that represents the tidal deformability of a matter body [23, 32-34]

$$
Q_{i j}=-\frac{2}{3} k_{2} \frac{R^{5}}{G} E_{i j},
$$

where $R$ is the Earth's mean radius. The tidal tensor $E_{i j}$ of the external field can be expressed in terms of the GW strain amplitude $\mathbf{h}$ in transverse traceless gauge by [35]

$$
E_{i j}=-\frac{1}{2} \ddot{h}_{i j}=\frac{\omega_{g}^{2}}{2} h_{i j}=\frac{2 \pi^{2} c^{2}}{\lambda_{g}^{2}} h_{i j},
$$

where the dot symbol means time derivative. Equation (9) is valid for static gravitational fields [34, 35]. This means that the time-scale of the variation of the external tidal field is much larger than the time-scale on which the deformed Earth rearranges its internal mass structure. The static approximation corresponds to the zero-frequency limit of Earth's dynamical response and can be applied at very low frequencies (below $0.3 \mathrm{mHz}$, the frequency of the lowest seismic mode). Hence we consider the long wavelength regime, meaning $\frac{2 \pi c}{\omega_{g}} \gg R$, where $\lambda_{g}=\frac{2 \pi c}{\omega_{g}}=\frac{c}{f_{g}}$ is the wavelength and $f_{g}$ the frequency of the $\mathrm{GW} \ll 0.3 \mathrm{mHz}$. In this condition we can also neglect the wave vector component in Equation (3) and write

$$
\mathbf{h}=h_{0} \epsilon e^{i \omega_{g} t}
$$


Here, we consider a right-hand circularly polarized GW in form $\boldsymbol{\epsilon}=\frac{1}{2}\left(\mathbf{e}_{+}-i \mathbf{e}_{\times}\right)$. We hence assume same proportion of each polarization. According to $[18,20]$, the right-hand circularly polarized tensor is defined in the Cartesian coordinate system $O=\left(\overrightarrow{e_{x}}, \overrightarrow{e_{y}}, \overrightarrow{e_{z}}\right)$ rotating with the Earth by

$$
\boldsymbol{\epsilon}=\frac{1}{2} e^{2 i \nu}\left(\begin{array}{ccc}
a_{1}^{2} & a_{1} a_{2} & -a_{1} a_{3} \\
a_{1} a_{2} & a_{2}^{2} & -a_{2} a_{3} \\
-a_{1} a_{3} & -a_{2} a_{3} & a_{3}^{2}
\end{array}\right)
$$

where

$$
\left\{\begin{array}{l}
a_{1}=\cos e \cos \lambda+i \sin \lambda \\
a_{2}=\cos e \sin \lambda-i \cos \lambda \\
a_{3}=\sin e
\end{array}\right.
$$

The components of a pure circularly polarized tensor plane wave approaching the Earth with a normal $\vec{p}$ in $O$ are hence $(\sin e \cos \lambda, \sin e \sin \lambda, \cos e)$. Perturbations in the quadrupole moment of the Earth can finally be written

$$
Q_{i j}=-k_{2} \frac{R^{5}}{G} \frac{2 \pi^{2} c^{2}}{3 \lambda_{g}^{2}} h_{0} e^{i \omega_{g} t} e^{2 i \nu}\left(\begin{array}{ccc}
a_{1}^{2} & a_{1} a_{2} & -a_{1} a_{3} \\
a_{1} a_{2} & a_{2}^{2} & -a_{2} a_{3} \\
-a_{1} a_{3} & -a_{2} a_{3} & a_{3}^{2}
\end{array}\right)
$$

or

$$
Q_{i j}=-k_{2} \frac{R^{5}}{G} \frac{\omega_{g}^{2}}{6} h_{0} e^{i \omega_{g} t} e^{2 i \nu}\left(\begin{array}{ccc}
a_{1}^{2} & a_{1} a_{2} & -a_{1} a_{3} \\
a_{1} a_{2} & a_{2}^{2} & -a_{2} a_{3} \\
-a_{1} a_{3} & -a_{2} a_{3} & a_{3}^{2}
\end{array}\right)=-c_{0} h_{0} \omega_{g}^{2} e^{i \omega_{g} t} f(e, \lambda, \nu)
$$

where we have introduced the time-independent parameter $c_{0}=k_{2} \frac{R^{5}}{6 G} \approx 7.8610^{42} \mathrm{~m}^{2} \mathrm{~kg} \mathrm{~s}{ }^{2}$ and the function

$$
f(e, \lambda, \nu)=e^{2 i \nu}\left(\begin{array}{ccc}
a_{1}^{2} & a_{1} a_{2} & -a_{1} a_{3} \\
a_{1} a_{2} & a_{2}^{2} & -a_{2} a_{3} \\
-a_{1} a_{3} & -a_{2} a_{3} & a_{3}^{2}
\end{array}\right),
$$

is defined by the incident angles of the GW. This function describes the geometrical shape of the deformation. Please note that all the components of the quadrupole moment $Q$ have same time dependency but different amplitudes. The maximum value of component $a_{1} a_{3}$ is for $\nu=0, \lambda=\pi / 2$ and $e=\pi / 3$. In such a case, the global Earth's deformation is characterized by a dilatation along $\vec{e}_{z}\left(a_{3}^{2}=\frac{3}{4}\right)$ and to a smaller extent along $\vec{e}_{y}\left(a_{2}^{2}=\frac{1}{4}\right)$ and a contraction along $\vec{e}_{x}\left(a_{1}^{2}=-1\right)$. When the $\mathrm{GW}$ is coming along the axial direction $(e=0$ or $\pi)$, then $a_{3}=0$, there would be no perturbation of the components $c_{i 3}$ of the inertia tensor and then no perturbation of the Earth's rotation. We also see that the amplitude of the perturbation of the momentum of inertia is proportional to the square of the GW frequency and to its strain amplitude.

\section{DYNAMICAL EQUATIONS OF THE EARTH'S ROTATION}

The Earth is rotating steadily at the rate $\Omega$ about the Earth's figure axis $\vec{e}_{z}$, fixed in inertial space (see Fig. 1). The two equatorial unit vectors $\vec{e}_{x}$ and $\vec{e}_{y}$ are orientated along the Earth's mean axes of inertia.

The action of any external torque or any mass redistribution within or at the surface of the Earth will perturb the Earth's rotation. We note $\vec{\omega}$ the instantaneous rotation vector, which coordinates in the steadily rotating terrestrial frame are defined as $\vec{\omega}=\vec{\Omega}\left(m_{1}, m_{2}, 1+m_{3}\right)$. The quantities $\left(m_{1}, m_{2}, 1\right)$ are the direction cosines of the rotation axis. The two principal equatorial directions ( $m_{1}$ is in the direction of Greenwich and $m_{2}$ is in the direction of $90^{\circ} \mathrm{E}$ ) define the variations in polar motion and $m_{3}$ the spin rate changes.

The conservation of the angular momentum $\vec{H}$ is written

$$
\dot{\vec{H}}+\vec{\omega} \wedge \vec{H}=0,
$$

in the rotating frame attached to the Earth and in the absence of any external torque acting on the body. The vector $\vec{H}$ can be expressed as a function of the tensor of inertia introduced previously in Eq. (5)

$$
H_{j}=I_{i j} \omega_{i} .
$$




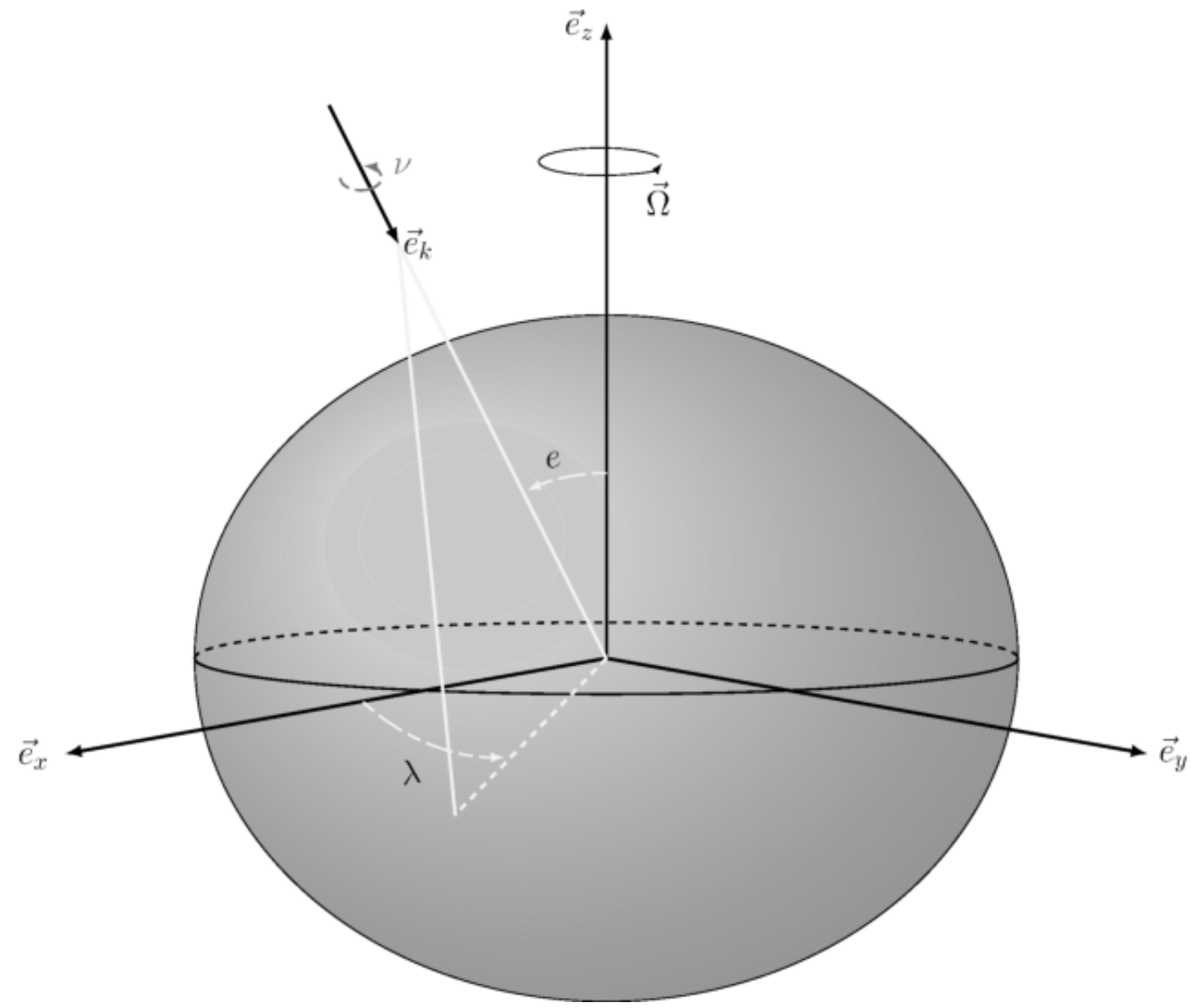

FIG. 1. Rotating terrestrial reference frame $O=\left(\vec{e}_{x}, \vec{e}_{y}, \vec{e}_{z}\right)$ and angles $e, \lambda, \nu$ defining the polarization plan of a gravitational wave.

After linearization and neglecting second-order quantities in $m_{i}$ and $c_{i j}$, the varying Earth's rotation is governed by the linearized form of the so-called Euler-Liouville equations [31, 36]

$$
\begin{array}{r}
\left(1-\frac{k_{2}}{k_{s}}\right) \tilde{m}+\frac{i}{\sigma_{e}} \dot{\tilde{m}}=\tilde{\Psi} \\
\dot{m}_{3}=\dot{\Psi}_{3},
\end{array}
$$

where $\sigma_{e}$ is the Eulerian nutation frequency $(=\alpha \Omega)$ and $\alpha=\frac{C-A}{A}$ is the dynamic ellipticity of the Earth. The frequency $\sigma_{C W}=\left(1-\frac{k_{2}}{k_{s}}\right) \sigma_{e}$ is the Chandler wobble frequency. The degree-2 $k_{2}$ and secular $k_{s}$ Love numbers characterize elastic deformations that take place under polar motion [37], which values are provided in Table I. The variations in the position of the Earth's rotation axis $\tilde{m}=m_{1}+i m_{2}$ is the Earth's polar motion. The second equation defines $m_{3}$ which is associated with the variation of length of day noted $\triangle L O D=-\overline{L O D} m_{3}$, where $\overline{L O D}$ is the nominal length of day, equal to $86400 \mathrm{~s}$. The function $\tilde{\Psi}=\Psi_{1}+i \Psi_{2}$ is the equatorial projection while $\Psi_{3}$ is the axial component of the excitation function. The excitation function $\Psi_{i}$ contains all possible geophysical effects on the rotation of the Earth, due to motion that carry angular momentum or to redistribution of mass, and to any external torque (tides, surficial fluid layers, etc...). The latter can be expressed in terms of changes in the non-diagonal elements $\tilde{c}=c_{13}+i c_{23}$,

$$
\begin{array}{r}
\tilde{\Psi}=\frac{\tilde{c}}{C-A}-\frac{i}{(C-A) \Omega} \dot{\tilde{c}}=\frac{\tilde{c}}{C-A}\left(1+\frac{\omega_{g}}{\Omega}\right) \\
\Psi_{3}=-\frac{c_{33}}{C} .
\end{array}
$$

The excitation functions defined in Eq.(17) represent the source terms of the perturbations of the Earth's rotation by GWs. 


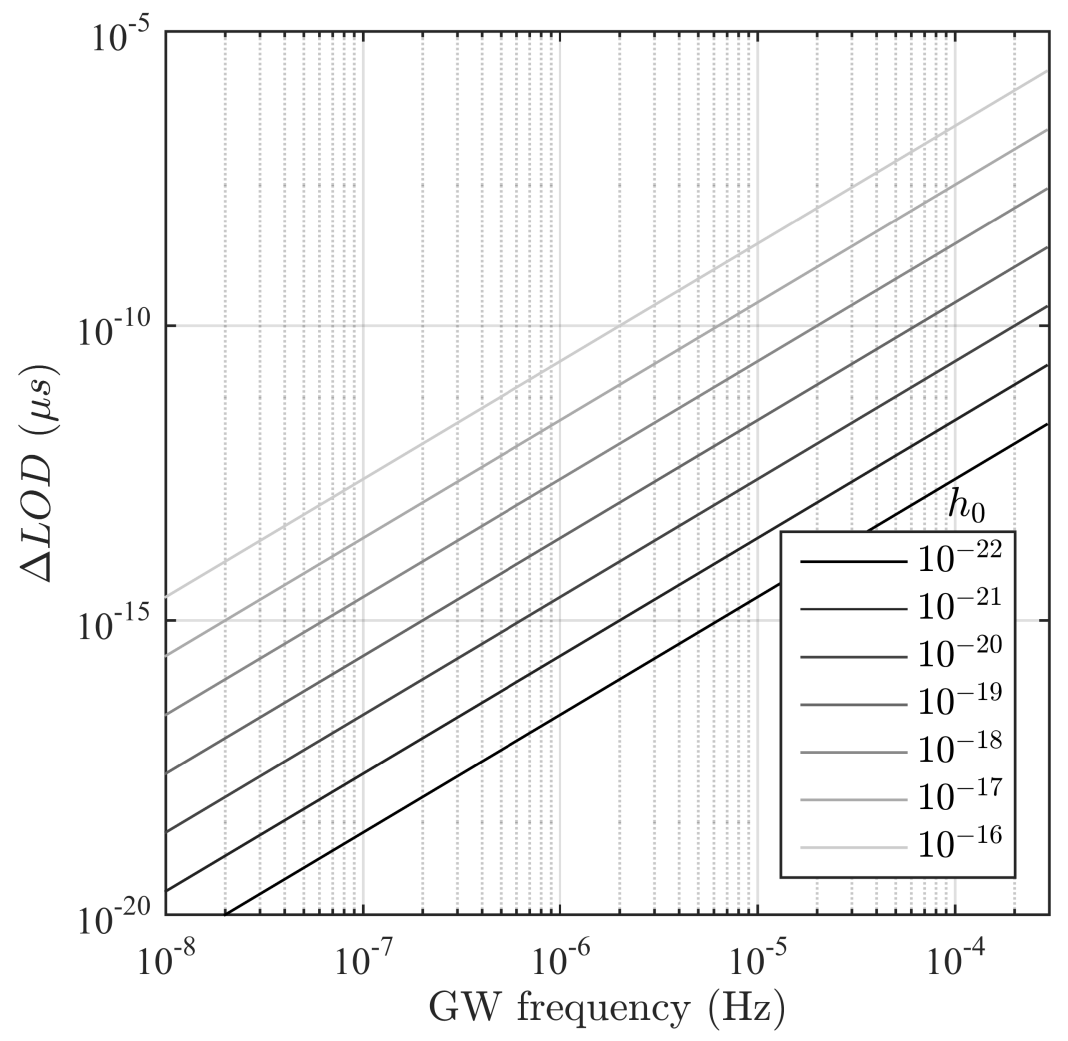

FIG. 2. Perturbation of the length of day of the Earth as a function of the frequency and for various amplitudes $h_{0}$ of the GW propagating at 60 degrees $(e=\pi / 3, \nu=0$ and $\lambda=\pi / 2)$ from the Earth's rotation axis.

\section{A. Amplitudes of the Earth's spin rate changes}

According to Eq. (16), the perturbation in the length of day induced by a GW is

$$
\frac{\Delta L O D}{\overline{L O D}}=\frac{c_{33}}{C}=\frac{c_{0}}{C} h_{0} \omega_{g}^{2} e^{i \omega_{g} t} e^{2 i \nu} \sin ^{2} e,
$$

where we recall that $e$ is the incident angle of the incoming GW with respect to the Earth's rotation axis as defined in Fig. 1. The perturbation of the Earth's spin rate is proportional to the square of the GW frequency, but we have to stay within the static field limit, so that the expected amplitude of the perturbation is limited. When $e=0$ or $\pi$, there is no increment of inertia along $\vec{e}_{z}$ and no perturbation of the axial rotation. When $e=\pi / 3$ and $\nu=0$, we would have a perturbation of the Earth's spin rate of $\sim 310^{6} f_{g}^{2} h_{0}$. The length of day change induced by a GW is plotted in Fig. 2 for various GW strain amplitudes. For a GW of frequency $f_{g}=10^{-4} \mathrm{~Hz}$, the wavelength $\lambda_{g} \approx 5$ $10^{5} R$ and the relative perturbation in the Earth's rotation rate would be $\sim 310^{-2} h_{0}$. For $h_{0}=10^{-16}$, we would expect an increment of inertia $c_{33}$ of $\sim 10^{-18} \mathrm{C}\left(\Delta L O D \sim 10^{-7} \mu \mathrm{s}\right)$ at $10^{-4} \mathrm{~Hz}$, that is 10 orders smaller than the zonal tidal contribution $c_{33} \sim 10^{-8} C[38]$.

\section{B. Solution for the excitation of polar motion}

The contributions to the inertia tensor from the mass redistribution of the Earth induced by a GW is obtained from Eq. (8) and (12)

$$
\tilde{c}=c_{0} h_{0} \omega_{g}^{2} e^{i \omega_{g} t} e^{2 i \nu} a_{3}\left(a_{1}+i a_{2}\right) .
$$

The amplitude of the associated source function $\tilde{\Psi}$ (Eq. (17)) is plotted in Fig. 3 for various GW strain amplitudes. When $e=0$ or $\pi$, there is no perturbation of the equatorial components of the rotation axis. Here also we take 
$e=\pi / 3$ and $\nu=0$. At $10^{-4} \mathrm{~Hz}$ and for $h_{0}=10^{-16}$, the relative inertia perturbation would be of the order of $\tilde{c} \sim 10^{-18} A$. At diurnal periods, it would be $\sim 10^{-20} A$ which would be considerably smaller than the contributions from tides of the order $\sim 10^{-8} A$ [37]. Earthquakes also perturb the Earth's tensor of inertia on minutes time-scales and are accompanied by variations of the order $\dot{c}_{i j} \approx 10^{-9} A \Omega[39]$. Here, at $10^{-4} \mathrm{~Hz}, \dot{c}_{i j} \approx 10^{-17} A \Omega$ for $h_{0}=10^{-16}$.

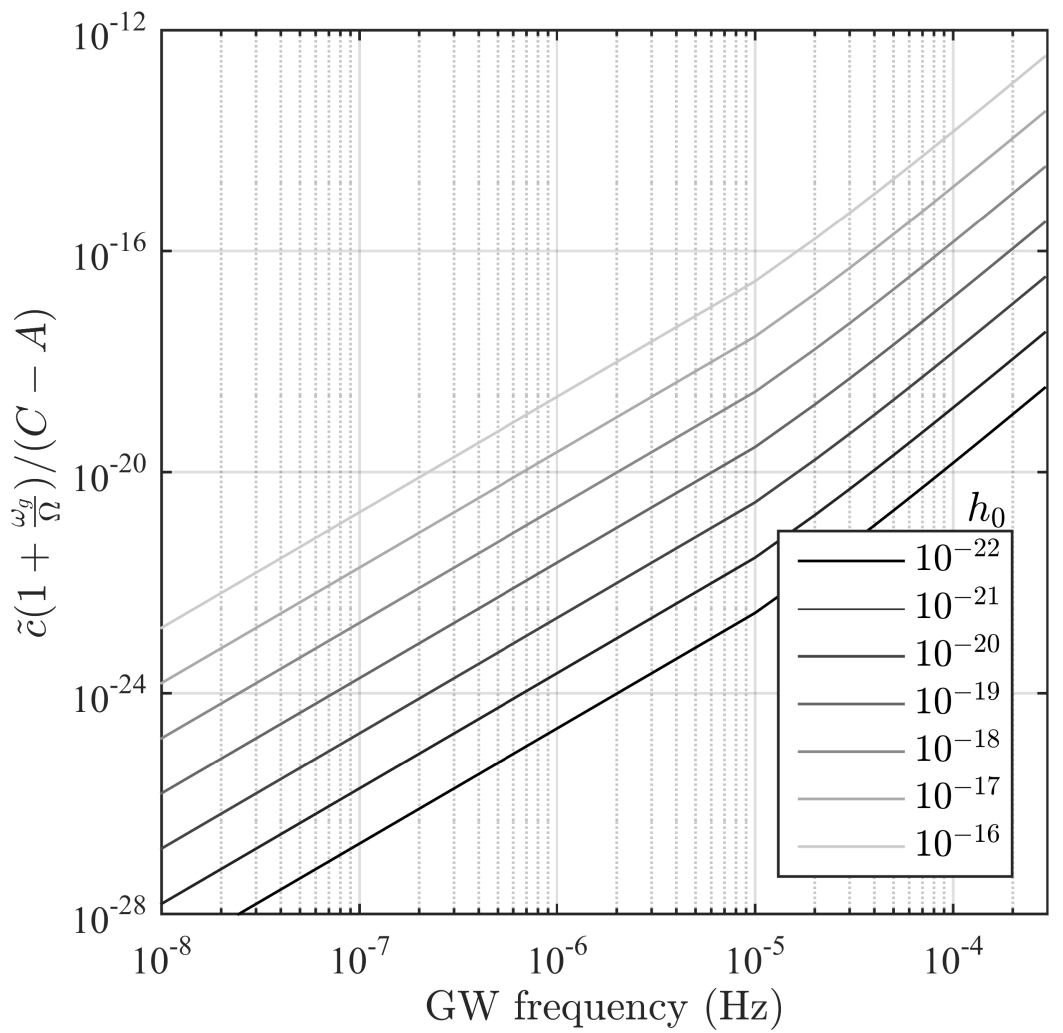

FIG. 3. Amplitude of the excitation term as a function of the frequency and for various amplitudes $h_{0}$ of the GW propagating at 60 degrees $(e=\pi / 3, \nu=0$ and $\lambda=\pi / 2)$ from the Earth's rotation axis. The angular frequency of the GW is $\omega_{g}$ and the Earth's rotation rate is noted $\Omega$. $A$ and $C$ are the principal inertial momentum of the Earth and $\tilde{c}$ is the perturbation of the inertia tensor of the Earth induced the GW.

The solution to Eq. (15) for the wobble $\tilde{m}$ due to any excitation function $\Psi$ is [31]

$$
\tilde{m}(\sigma)=-\frac{\sigma_{C W}}{\sigma-\sigma_{C W}} \Psi(\sigma),
$$

in frequency domain, or in time-domain

$$
\tilde{m}(t)=-i \sigma_{C W} e^{i \sigma_{C W} t} \int_{-\infty}^{t} \Psi(\tau) e^{-i \sigma_{C W} \tau} d \tau .
$$

Considering Eq. (17) and Eq. (18), for an infinite monochromatic wave, the source function is defined by

$$
\Psi(t)=\frac{c_{0} h_{0}}{A}\left(1+\frac{\omega_{g}}{\Omega}\right) \frac{\Omega}{\sigma_{e}} \omega_{g}^{2} e^{i \omega_{g} t} e^{2 i \nu} a_{3}\left(a_{1}+i a_{2}\right)=\Psi_{0} e^{i \omega_{g} t}
$$

and

$$
\Psi(\sigma)=\Psi_{0} \delta\left(\sigma-\omega_{g}\right),
$$

where we have defined $\Psi_{0}=\frac{c_{0} h_{0}}{A}\left(1+\frac{\omega_{g}}{\Omega}\right) \frac{\Omega}{\sigma_{e}} \omega_{g}^{2} e^{2 i \nu} a_{3}\left(a_{1}+i a_{2}\right)$. If we integrate from an initial time $t_{0}$, then the polar motion $\tilde{m}$ will be the sum of the free Chandler wobble with initial amplitude $m_{0}$ and a forced wobble at the angular frequency $\omega_{g}$

$$
\tilde{m}(t)=m_{0} e^{i \sigma_{C W}\left(t-t_{0}\right)}+\frac{\sigma_{C W}}{\sigma_{C W}-\omega_{g}} \Psi_{0} e^{i \omega_{g} t} .
$$




\begin{tabular}{l|c|l}
\hline Gravitational constant & $\mathrm{G}$ & $6.6740810^{-11} \mathrm{~m}^{3} \mathrm{~kg}^{-1} \mathrm{~s}^{-2}$ \\
Speed of light in vacuum & $\mathrm{c}$ & $299792458 \mathrm{~m} / \mathrm{s}$ \\
Earth's rotation rate & $\Omega$ & $7.29211510^{-5} \mathrm{rad} / \mathrm{s}$ \\
Earth's mean radius & $R$ & $6.37110^{6} \mathrm{~m}$ \\
Principal inertial momentum & $A$ & $8.010110^{37} \mathrm{~kg} \mathrm{~m}$ \\
Axial inertial momentum & $C$ & $8.036510^{37} \mathrm{~kg} \mathrm{~m}^{2}$ \\
Dynamic ellipticity & $\alpha$ & $3.284547910^{-3}$ \\
Earth's mean density & $\rho_{0}$ & $5500 \mathrm{~kg} \mathrm{~m}^{-3}$ \\
\hline Degree-2 Love number & $k_{2}$ & 0.302 \\
Secular Love number & $k_{s}$ & 0.942 \\
Degree-2 Shida number & $l$ & 0.085 \\
Degree-2 tidal gravimetric factor & $\delta$ & 1.16 \\
\hline
\end{tabular}

TABLE I. Numerical values of parameters for an Earth's PREM model [40] and taken from Petit and Luzum [41] and Mathews et al. [37].

From the second term in Eq. (21), we can compute the expected amplitudes for the polar motion excitation oscillating at the pulsation $\omega_{g}$ for various GW amplitudes and frequencies. Parameters values for the PREM [40] Earth's model are provided in Table I. When the GW frequency is close to the CW frequency, a resonance would occur. When considering the presence of the elliptical fluid core inside the Earth, a second rotational mode exists, that is the free core nutation (FCN) which period is nearly diurnal in the Earth's rotating frame. For GWs with a period close to one day, a resonance with the FCN mode would occur too. However, since diurnal tides are already forcing that mode, it would be impossible to distinguish a GW origin in geodetic observations.

\section{DISCUSSION}

\section{A. Detectability in terms of LOD variations}

The relative perturbation of the rotation rate of $10^{-18}$ obtained for a $10^{-4} \mathrm{~Hz} \mathrm{GW}$ with an amplitude $10^{-16}$ would be ten orders of magnitude smaller than the resolution of recent ring-laser gyroscopes of $\sim 10^{-8}$ [42]. Large ring laser gyroscope (like the G-ring) can reach a sensitivity of $1.210^{-11} \mathrm{rad} / \mathrm{s} / \sqrt{\mathrm{Hz}}$ [43], while sensitivity of the VLBI (very long baseline interferometry) is of the order $\sim 6 \mathrm{~ms} / \sqrt{\mathrm{Hz}}$ or $\sim 510^{-12} \mathrm{rad} / \mathrm{s} / \sqrt{\mathrm{Hz}}[44]$, both being characterized by white noise. Besides a weak amplitude, the presence of larger LOD variations of geophysical origin would mask the GW effect. Amplitude spectral densities of geophysical (atmosphere, oceans, hydrosphere, fluid core) contributions to the variations of LOD and the observed LOD variations corrected for zonal tides show that the GW effect would indeed be elusive but with an amplitude increasing with the frequency (Fig. 4). Observed LOD variations were downloaded from the International Earth Rotation and Reference Systems Service (IERS) (http://hpiers.obspm.fr). Atmospheric, oceanic and hydrological angular momentia were downloaded from the ESMGFZ Product Repository generated by the Earth-System-Modelling group [45]. Core angular momentum contribution was obtained from https://geodyn.univ-grenoble-alpes.fr/ [46].

\section{B. Detectability in polar motion observations}

The observed amplitude of the CW excited mostly by surficial fluid layers (mostly atmosphere and oceans) is of the order of a few milliarcseconds [47] in terms of relative polar motion $\tilde{m}$ components where 1 arcsecond $=2 \pi / 360 / 3600$ radians. Even with a large fluctuation of the space-time with a strain amplitude $h_{0}=10^{-16}$, the amplitude of the induced polar motion at $10^{-4} \mathrm{~Hz}$ would be $\sim 10^{-9}$ mas, that is to say, nearly ten orders of magnitude smaller.

On Fig. 5 the sensitivity curves of the VLBI and GNSS (Global Navigation Satellite System) used by the IERS to retrieve polar motion observations show that the GW effect is at least five orders of magnitude below the levels of detectability of these techniques. The VLBI sensitivity is characterized by a white noise of variance $10^{5} \mu \mathrm{as}^{2}$ integrated over one day. For the GNSS, the amplitude spectral density can be defined by $\frac{\sigma_{a}}{\sqrt{4 \ln (2) f}}$, where $\sigma_{a}=60 \mu$ as is the Allan variance and $f$ the frequency $[48,49]$. This empirical formula is valid in the range between 2 and 1000 days. For shorter periods, the amplitude spectral density should be larger but below a white noise with amplitude 11 mas $/ \sqrt{\mathrm{Hz}}$ 
[49]. The sensitivity of the GNSS improves with the frequency till 2 days but is still beyond the detectability level for large GW events (i.e. with $h_{0}=10^{-16}$, Fig. 5). The amplitude of the GW-induced polar motion is also below the sensitivity level of VLBI. Current estimates of the Earth rotation parameters are limited at 2-day periods even if some attempts have been made to estimate sub-daily rotation parameters [50]. However processing of the geodetic data analysis significantly affects the resulting Earth rotation parameters, in particular in the shortest periods [51]. Progress still needs to be made to reach the high-frequency sensitivity required to be able to detect GWs through their effects on polar motion.

\section{Detectability through centrifugal deformation}

The centrifugal potential associated with polar motion induces surface deformation with horizontal displacement approximately defined as $l \frac{\Omega^{2} R^{2}}{g}|\tilde{m}| \approx 1.910^{3}|\tilde{m}| \mathrm{m}[31,52]$, where $l=0.085$ is the second-order body tide displacement Shida number and $|\tilde{m}|$ is given in radians. The relative strain perturbation is $\approx 2 \times 1.910^{3}|\tilde{m}| / R$. This is a rough estimate of the strain amplitude that we have represented on the right-hand axis on Fig. 5 . This centrifugal deformation associated with a GW is beyond the sensitivity range of current GW detectors but would be within the sensitivity range of the future LISA mission [53]. Induced gravity perturbation would be roughly $\Delta g=\delta \Omega^{2} R|\tilde{m}| \approx 3.910^{6}|\tilde{m}|$ $\mu \mathrm{Gal}$, where $\delta=1.16$ is the second-order body tide gravimetric factor. For a polar motion of $10^{-10}$ mas, i.e. $510^{-19}$ rad, $\Delta g=210^{-12} \mu \mathrm{Gal}$ which is elusive and well underneath detectability. Effet of $m_{3}$ (LOD variations) would be even smaller [52]. In the above strain and gravity estimates, we have neglected latitude dependencies. Horizontal displacements are indeed marginally important at high and low latitudes but are utterly negligible at mid-latitudes [52]. So the strain values proposed here are maximum amplitudes.

Please note that the total increment of inertia would be the sum of the direct effect from the tidal field (the GW effect) and the indirect centrifugal effect arising from Earth's rotation variations (the centrifugal deformation).

\section{On the Earth's deformability}

The deformation of the Earth in response to an external tidal field is represented by the Love number $k_{2}$ which value is typically around 0.3 for an elastic Earth's model [54] or as inferred from gravimetric observations [e.g. 55]. This potential Love number is however frequency dependent $[33,56]$. When taking into account the influence of the oceans, its equilibrium response leads to $k_{2} \sim 0.35$ at the frequency of CW. But, for daily and sub-daily frequencies, as the ocean response to tidal excitation becomes dynamical, $k_{2}$ is strongly affected, especially its imaginary part, getting as large as the real part. One of the consequences is a decrease of the resonance period of the polar motion, dropping from 432 down to 400 days and less [39]. More generally the higher the excitation frequency, the smaller is the deformational response, as the Earth tends to rigidify. Our estimates represent then an upper limit of the expected effects of GWs on the Earth's tensor of inertia and rotation.

\section{E. On the GW source model}

We have considered a simple waveform model for the GW with same proportion of each polarization $\mathbf{e}_{+}$and $\mathbf{e}_{\times}$that contributes to the signal [Eq. (11)] and represented by an infinite monochromatic wave. Such an approximation is valid for some identified double white dwarfs systems. In a more general way, the monochromatic wave approximation is valid for a continuous GW source like for instance a single spinning massive object or detached double white dwarfs. The later vastly outnumber all other compact binary objects in the Galactic disk [57] and their properties are constant in time. Their amplitude is however smaller than other cataclysmic events. For identified double binary white-dwarfs $h_{0} \sim 10^{-22}[57]$ which would result in polar motion and length of day variations clearly beyond the detectability level of current instruments (Fig. 4 and Fig. 5). For wavebursts like supernovae explosions a Gaussian or sine-Gaussian wave model should be used with periods less than one second. With bursts sources, we are however beyond the limit of a static gravitational field as used in this study. For other transient GW events like binary black holes that cannot be modeled by a specific waveform, a more complex numerical model that describes the polarization tensor $\epsilon$ very accurately should be employed [2]. The detector response should also be incorporated for an accurate estimate [58]. This is however beyond the scope of this paper that aims at providing some first estimates of the GW effect on the Earth's rotation for a continuous monochromatic waveform. 


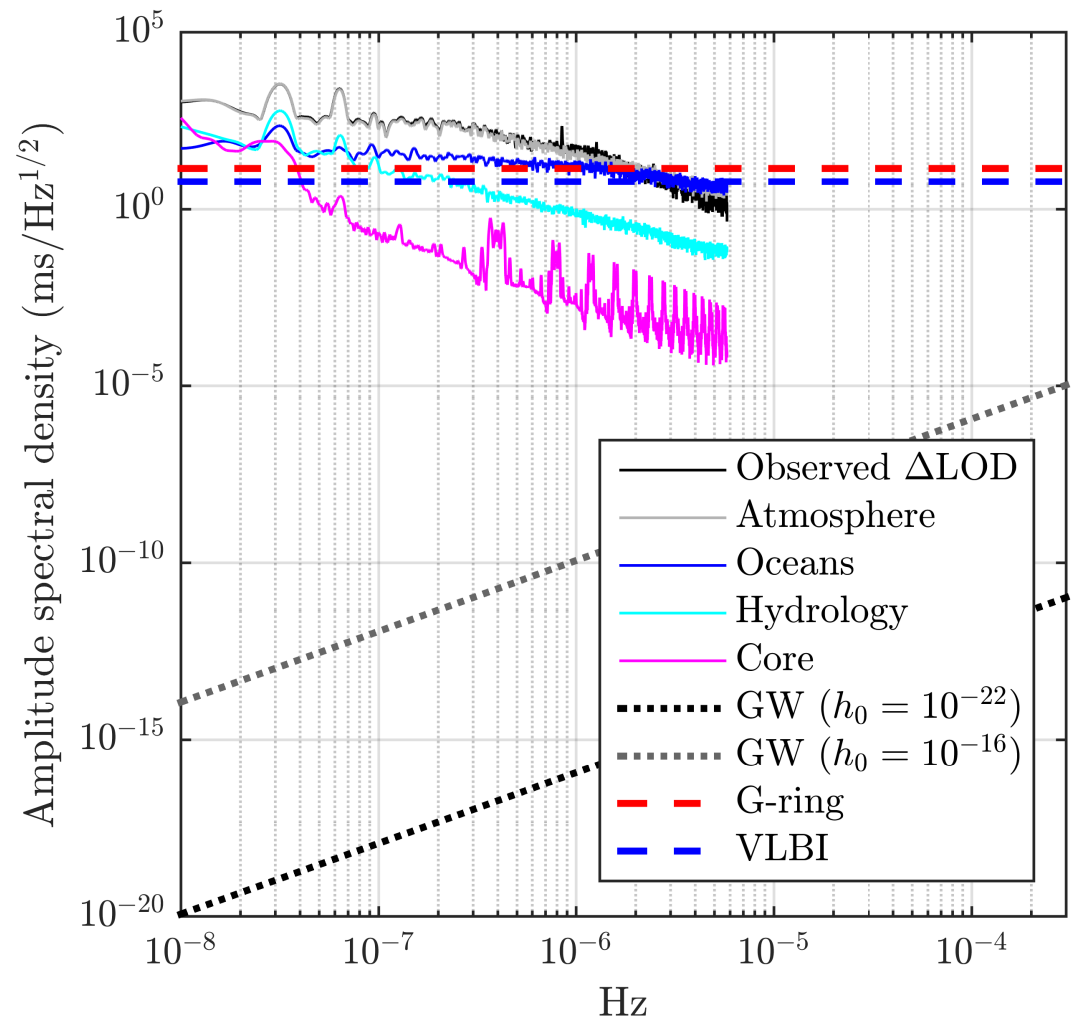

FIG. 4. Amplitude spectral densities of the observed (after correcting for zonal tides) and predicted variations of length of day $(\triangle L O D)$ for the main geophysical sources (atmosphere, oceans, continental hydrology and core angular momentum) and for GWs with amplitudes $h_{0}=10^{-22}$ and $10^{-16}$ (black and gray dotted lines). The sensitivity of the large laser-ring gyroscope (G-ring [43]) is indicated in red dashed line and for the VLBI [49] in dashed blue line.

\section{CONCLUSION}

We have employed an angular momentum approach considering the action of gravitational waves from astrophysical sources acting on an axisymmetric ellipsoidal Earth's model. We have shown that space-time fluctuations of astrophysical origin could affect the Earth's rotation through the perturbation of its moments of inertia. The maximum perturbation of the tensor of inertia induced by a monochromatic GW of frequency $f_{g}$ reaching the Earth would be of the order of $10^{14} f_{g}^{2} h_{0}$, where $h_{0}$ is the strain amplitude of the space-time fluctuation associated with a GW. The perturbation of the spin rate and of the equatorial components of the polar motion would be ten to fifteen orders of magnitude smaller than the observed excitation by surficial fluid layers (atmosphere, oceans, hydrology). A resonance effect with the rotational modes of the Earth could in principle occur but the associated resonance strengths would be small and hidden in the existing excitation by Earth's surficial layers. At frequencies higher than the resonance with the Chandler wobble, the polar motion excited by GWs would be beyond the detectablity range of GNSS and VLBI sensors. Current estimates of the Earth rotation parameters are however limited at 2-day periods. Progress still needs to be made to reach the sensitivity required to be able to detect GWs through their effects on polar motion. The centrifugal deformation associated with the GW-induced polar motion would also be beyond the sensitivity of the laser strainmeters used to detect GWs but could be within the sensitivity range of the future LISA antenna. We have employed a static limit approximation to investigate the effect of GWs on the Earth's rotation. In order to extend our work at seismic frequencies, we would need a normal mode formalism as used in Majstorović et al. [20]. We have shown that using the Earth's rotation as a detector of GWs would not be currently efficient. Our work could however be useful in the future when instrument sensitivities to measure the Earth's rotation improve. 


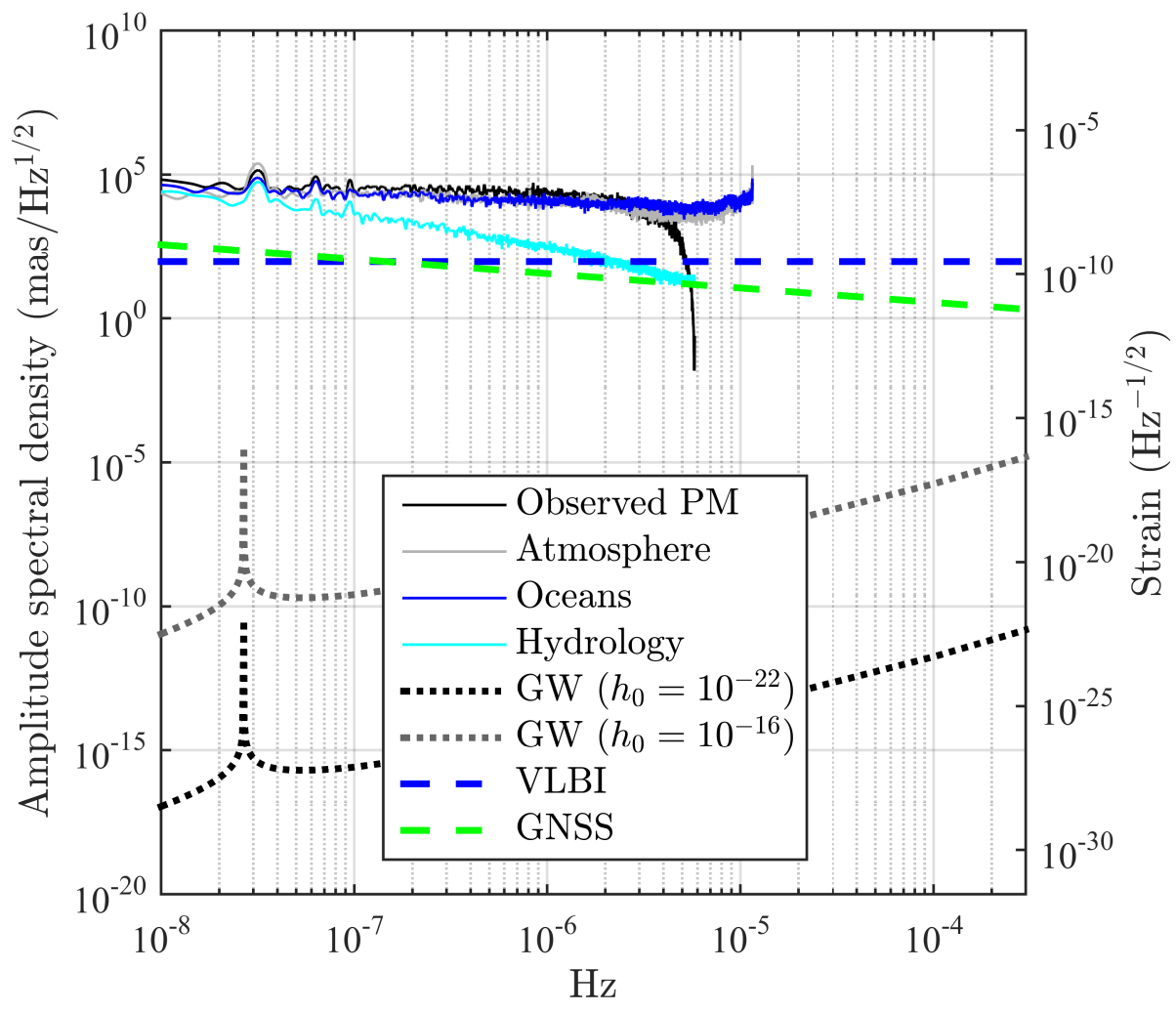

FIG. 5. Amplitude spectral densities of the observed (after correcting for Chandler and annual wobbles) and predicted equatorial polar motion (PM) for the main geophysical sources (atmosphere, oceans and continental hydrology). The amplitudes of polar motion induced by GWs with amplitudes $h_{0}=10^{-22}$ and $10^{-16}$ are plotted in black and gray dotted lines. VLBI and GNSS sensitivities (from [49]) are respectively plotted in dashed blue and green lines. Above $10^{-5} \mathrm{~Hz}$, the GNSS sensitivity curve in terms of polar motion is unknown, so only a lower bound is plotted. The peak around $2.710^{-8} \mathrm{~Hz}$ corresponds to the resonance with Chandler wobble.

\section{ACKNOWLEDGMENTS}

S.R. wants to thank Jérôme Pétri (Observatory of Strasbourg, France) and Jan Harms (GSSI, Italy) for some fruitful discussions about this work. We also thank Christian Bizouard (Observatory of Paris, France) for a deep reading and constructive suggestions as well as an anonymous reviewer for major comments to improve this work. This study was supported by the Programme National de Planétologie (PNP) of CNRS/INSU co-funded by CNES.

[1] B. P. Abbott et al. (The LIGO Scientific Collaboration and The Virgo Collaboration), Observation of gravitational waves from a binary black hole merger, Physical Review Letter 116, 061102 (2016).

[2] B. Abbott, R. Abbott, T. Abbott, S. Abraham, F. Acernese, K. Ackley, C. Adams, R. Adhikari, V. Adya, C. Affeldt, and et al., GWTC-1: A Gravitational-Wave Transient Catalog of Compact Binary Mergers Observed by LIGO and Virgo during the First and Second Observing Runs, Physical Review X 9, 10.1103/physrevx.9.031040 (2019).

[3] B. P. Abbott et al., GWTC-2: Compact Binary Coalescences Observed by LIGO and Virgo During the First Half of the Third Observing Run (2020), arXiv:2010.14527 [gr-qc].

[4] J. Weber, Detection and generation of gravitational waves, Physical Review 117, 306 (1959).

[5] G. Pizzella, A brief review of the gravitational wave resonant detectors, Nuclear Physics B - Proceedings Supplements 35 , 71 (1994).

[6] G. Frossati, Ultralow-temperature resonant gravitational wave detectors, present state and future prospects, Advances in Space Research 32, 1227 (2003), fundamental Physics in Space.

[7] F. Ronga, Detection of gravitational waves with resonant antennas, Journal of Physics: Conference Series 39, 18 (2006). 
[8] A. D. Waard, L. Gottardi, J. V. Houwelingen, A. Shumack, and G. Frossati, MiniGRAIL, the first spherical detector, Classical and Quantum Gravity 20 (2003).

[9] O. D. Aguiar et al., The status of the Brazilian spherical detector, Classical and Quantum Gravity 19 (2002).

[10] P. Bender, I. Ciufolini, K. Danzmann, W. Folkner, J. Hough, D. Robertson, A. Rüdiger, M. Sandford, R. Schilling, B. Schutz, R. Stebbins, T. Sumner, P. Touboul, S. Vitale, H. Ward, W. Winkler, J. Cornelisse, F. Hechler, Y. Jafry, and R. Reinhard, LISA. Laser Interferometer Space Antenna for the detection and observation of gravitational waves. A Cornerstone Project in ESA's Long Term Space Science Programme "Horizon 2000 Plus", Pre-Phase A Report (1995).

[11] T. Shimoda, S. Takano, C. P. Ooi, N. Aritomi, Y. Michimura, M. Ando, and A. Shoda, Torsion-bar antenna: A groundbased mid-frequency and low-frequency gravitational wave detector, International Journal of Modern Physics D 29, 1940003 (2019).

[12] H. J. Paik, C. E. Griggs, M. V. Moody, K. Venkateswara, H. M. Lee, A. B. Nielsen, E. Majorana, and J. Harms, Lowfrequency terrestrial tensor gravitational-wave detector, Classical and Quantum Gravity 33, 075003 (2016).

[13] B. Canuel, A. Bertoldi, L. Amand, E. Pozzo di Borgo, T. Chantrait, C. Danquigny, M. Dovale Álvarez, B. Fang, A. Freise, R. Geiger, J. Gillot, S. Henry, J. Hinderer, D. Holleville, J. Junca, G. Lefèvre, M. Merzougui, N. Mielec, T. Monfret, S. Pelisson, M. Prevedelli, S. Reynaud, I. Riou, Y. Rogister, S. Rosat, E. Cormier, A. Landragin, W. Chaibi, S. Gaffet, and P. Bouyer, Exploring gravity with the MIGA large scale atom interferometer, Scientific Reports 8, 14064 (2018).

[14] C. J. Moore, The sensitivity of pulsar timing arrays, Journal of Physics: Conference Series 610, 012018 (2015).

[15] J. Dyson, F., Seismic response of the Earth to a gravitational wave in the 1-Hz band, The Astrophysical Journal 156, 529 (1969).

[16] V. S. Tuman, A search for gravity waves by means of the earth eigen vibrations, General Relativity and Gravitation 4, 279 (1973).

[17] O. G. Jensen, Seismic detection of gravitational radiation, Reviews of geophysics and space physics 17, 2057 (1979).

[18] A. Ben-Menahem, Excitation of the Earth's eigenvibrations by gravitational radiation from astrophysical sources, II Nuovo Cimento C 6, 49 (1983).

[19] M. Coughlin and J. Harms, Constraining the gravitational wave energy density of the Universe using Earth's ring, Phys. Rev. D 90, 042005 (2014).

[20] J. Majstorović, S. Rosat, and Y. Rogister, Earth's spheroidal motion induced by a gravitational wave in flat spacetime, Phys. Rev. D 100, 044048 (2019).

[21] J. Majstorović, S. Rosat, and Y. Rogister, Erratum: Earth's spheroidal motion induced by a gravitational wave in flat spacetime [phys. rev. d 100, 044048 (2019)], Phys. Rev. D 103, 029901 (2021).

[22] C. W. Misner, K. S. Thorne, and J. A. Wheeler, Gravitation (W. H. Freeman and Company, 1973).

[23] E. Poisson and C. M. Will, Gravity: Newtonian, Post-Newtonian, Relativistic (Cambridge University Press, 2014).

[24] M. Coughlin and J. Harms, Upper Limit on a Stochastic Background of Gravitational Waves from Seismic Measurements in the Range 0.05-1 Hz, Phys. Rev. Lett. 112, 101102 (2014).

[25] M. Coughlin and J. Harms, Constraining the gravitational-wave energy density of the Universe in the range $0.1 \mathrm{~Hz}$ to 1 $\mathrm{Hz}$ using the Apollo Seismic Array, Phys. Rev. D 90, 102001 (2014).

[26] J. Anandan and R. Chiao, Gravitational radiation antennas using the Sagnac effect, General Relativity and Gravitation 14, 515 (1982).

[27] J. Frauendiener, Gravitational waves and the Sagnac effect, Classical and Quantum Gravity 37, 05LT01 (2020).

[28] J. Nastula and R. Gross, Chandler wobble parameters from SLR and GRACE, Journal of Geophysical Research: Solid Earth 120, 4474 (2015).

[29] C. J. Moore, R. H. Cole, and C. P. L. Berry, Gravitational-wave sensitivity curves, Classical and Quantum Gravity 32 (2015).

[30] S. Rosat and S. B. Lambert, Free core nutation resonance parameters from VLBI and superconducting gravimeter data, Astronomy and Astrophysics - A\&A 503, 287 (2009).

[31] W. Munk and G. MacDonald, The Rotation of the Earth: a Geophysical Discussion (Cambridge Univ. Press, 1960).

[32] A. Love, Some Problems of Geodynamics (Cambridge University Press, 1911).

[33] J. M. Wahr, Body tides on an elliptical, rotating, elastic and oceanless earth, Geophysical Journal International 64, 677 (1981), http://oup.prod.sis.lan/gji/article-pdf/64/3/677/1780435/64-3-677.pdf.

[34] T. Hinderer, Tidal love numbers of neutron stars, The Astrophysical Journal 677, 1216 (2008).

[35] A. Das, S. S. Dave, O. Ganguly, and A. M. Srivastava, Pulsars as Weber gravitational wave detectors, Physics Letters B 791, 167 (2019).

[36] K. Lambeck, The Earth's Variable Rotation: Geophysical Causes and Consequences, Cambridge Monographs on Mechanics (Cambridge University Press, 1980).

[37] P. M. Mathews, T. A. Herring, and B. A. Buffett, Modeling of nutation and precession: New nutation series for nonrigid Earth and insights into the Earth's interior, Journal of Geophysical Research: Solid Earth 107, ETG 3 (2002).

[38] P. Defraigne and I. Smits, Length of day variations due to zonal tides for an inelastic earth in non-hydrostatic equilibrium, Geophysical Journal International 139, 563 (1999).

[39] C. Bizouard, I. Nurul Huda, Y. Ziegler, and S. Lambert, Frequency dependence of the polar motion resonance, Geophysical Journal International 220, 753 (2020).

[40] A. M. Dziewonski and D. L. Anderson, Preliminary reference Earth model, Physics of the Earth and Planetary Interiors 25, 297 (1981). 
[41] G. Petit and B. Luzum, eds., IERS Conventions (2010) (IERS Technical Note 36) (Frankfurt am Main: Verlag des Bundesamts für Kartographie und Geodäsie, 2010).

[42] R. B. Hurst, M. Mayerbacher, A. Gebauer, K. U. Schreiber, and J.-P. R. Wells, High-accuracy absolute rotation rate measurements with a large ring laser gyro: establishing the scale factor, Appl. Opt. 56, 1124 (2017).

[43] K. U. Schreiber, T. Klügel, J.-P. R. Wells, R. B. Hurst, and A. Gebauer, How to Detect the Chandler and the Annual Wobble of the Earth with a Large Ring Laser Gyroscope, Phys. Rev. Lett. 107, 173904 (2011).

[44] M. Karbon, B. Soja, T. Nilsson, Z. Deng, R. Heinkelmann, and H. Schuh, Earth orientation parameters from VLBI determined with a Kalman filter, Geodesy and Geodynamics 8, 396 (2017), Geodesy, Astronomy and Geophysics in Earth Rotation.

[45] H. Dobslaw and R. Dill, Predicting Earth orientation changes from global forecasts of atmosphere-hydrosphere dynamics, Advances in Space Research 61, 1047 (2018).

[46] N. Gillet, D. Jault, and C. C. Finlay, Planetary gyre, time-dependent eddies, torsional waves, and equatorial jets at the Earth's core surface, Journal of Geophysical Research: Solid Earth 120, 3991 (2015).

[47] Bizouard, C., Remus, F., Lambert, S. B., Seoane, L., and Gambis, D., The Earth's variable Chandler wobble, A\&A 526, A106 (2011).

[48] C. Bizouard, S. Lambert, C. Gattano, O. Becker, and J.-Y. Richard, The IERS EOP 14C04 solution for Earth orientation parameters consistent with ITRF 2014, Journal of Geodesy 93, 621 (2019).

[49] C. Bizouard, Geophysical Modelling of the Polar Motion (De Gruyter, Berlin, Boston, 2020).

[50] A. E. Sibois, S. D. Desai, W. Bertiger, and B. J. Haines, Analysis of decade-long time series of GPS-based polar motion estimates at 15-min temporal resolution, Journal of Geodesy 91, 965 (2017).

[51] R. Dill, H. Dobslaw, H. Hellmers, A. Kehm, M. Bloßfeld, M. Thomas, F. Seitz, D. Thaller, U. Hugentobler, and E. Schönemann, Evaluating Processing Choices for the Geodetic Estimation of Earth Orientation Parameters With Numerical Models of Global Geophysical Fluids, Journal of Geophysical Research: Solid Earth 125, 10.1029/2020JB020025 (2020).

[52] J. M. Wahr, Deformation Induced by Polar Motion, Journal of Geophysical Research 90, 9363 (1985).

[53] P. Amaro-Seoane, H. Audley, S. Babak, J. Baker, E. Barausse, P. Bender, E. Berti, P. Binétruy, M. Born, D. Bortoluzzi, J. Camp, C. Caprini, V. Cardoso, M. Colpi, J. Conklin, N. Cornish, C. Cutler, K. Danzmann, R. Dolesi, L. Ferraioli, V. Ferroni, E. Fitzsimons, J. Gair, L. Boté, D. Giardini, F. Gibert, C. Grimani, H. Halloin, G. Heinzel, T. Hertog, M. Hewitson, K. Holley-Bockelmann, D. Hollington, M. Hueller, H. Inchauspe, P. Jetzer, N. Karnesis, C. Killow, A. Klein, B. Klipstein, N. Korsakova, S. Larson, J. Livas, I. Lloro, N. Man, D. Mance, J. Martino, I. Mateos, K. McKenzie, S. Mcwilliams, C. Miller, G. Mueller, G. Nardini, G. Nelemans, M. Nofrarías, A. Petiteau, P. Pivato, E. Plagnol, E. Porter, J. Reiche, D. Robertson, N. Robertson, E. Rossi, G. Russano, B. Schutz, A. Sesana, D. Shoemaker, J. Slutsky, C. Sopuerta, T. Sumner, N. Tamanini, I. Thorpe, M. Tröbs, M. Vallisneri, A. Vecchio, D. Vetrugno, S. Vitale, M. Volonteri, G. Wanner, H. Ward, P. Wass, W. Weber, J. Ziemer, and P. Zweifel, Laser interferometer space antenna (2012).

[54] I. Longman, Computation of Love numbers and load deformation coefficients for a model Earth, Geophys. J. R. Astron. Soc. 11, 133 (1966).

[55] R. D. Ray, E. Richard J., and L. Frank G., Constraints on energy dissipation in the Earth's body tide from satellite tracking and altimetry, Geophys. J. Int. 144, 471 (2001).

[56] S. R. Dickman, Rotationally consistent Love numbers, Geophysical Journal International 161, 31 (2005).

[57] G. Nelemans, L. R. Yungelson, and S. F. Portegies Zwart, The gravitational wave signal from the Galactic disk population of binaries containing two compact objects, Astronomy \& Astrophysics 375, 890 (2001).

[58] M. Maggiore, Gravitational Waves: Volume 1: Theory and Experiments (Oxford University Press, 2007). 